1 Universidade Estadual de Campinas (Unicamp) Campinas (SP), Brasil. fernandes.juliana@gmail. com

2 Universidade Estadual de Campinas (Unicamp) Faculdade de Ciências Médicas - Campinas (SP), Brasil.

\section{Perfil das gestantes de alto risco e a cogestão da decisão sobre a via de parto entre médico e gestante}

\author{
Profile of high-risk pregnant women and co-management of the \\ decision on the routh of birth delivery between doctor and pregnant \\ woman
}

Juliana Azevedo Fernandes', Gastão Wagner de Sousa Campos², Priscila Maria Stolses Bergamo Francisco 2

DOI: $10.1590 / 0103-1104201912109$

RESUMO Estudo transversal realizado com dados de inquérito de serviços em quatro grandes cidades brasileiras. Esta investigação analisa dados de Campinas (SP), objetivando caracterizar o perfil das gestantes de alto risco acompanhadas nos ambulatórios públicos especializados, bem como verificar e discutir aspectos relacionados ao cuidado compartilhado na decisão da via de parto durante o pré-natal especializado. Amostra calculada considerando a prevalência de gestação de alto risco, perfil de morbidade e cobertura dos serviços especializados. Questionário aplicado a 405 gestantes desses serviços, e realizada análise descritiva a partir da distribuição de frequências das questões. Perfil de gestantes majoritariamente jovens, negras, com ensino médio completo e sem plano de saúde. Verificou-se associação estatística entre variáveis: quando as mulheres fazem a escolha do seu parto individualmente, a maioria opta pelo parto normal; quando apenas o médico decide, a maioria indica cesariana; e, quando se decide conjuntamente, prevalece a cesariana, porém, em percentual mais baixo do que quando o médico decide sozinho. Destaca-se a necessidade de discutir a assimetria de poder entre médicos e usuários na cogestão do cuidado como componente fundamental para qualificar o trabalho em saúde.

PALAVRAS-CHAVE Cuidado pré-natal. Gravidez de alto risco. Assistência integral à saúde. Tomada de decisões. Autonomia pessoal.

ABSTRACT Cross-sectional study carried out with survey data in four large Brazilian cities. This research analyzes data from Campinas (SP), aiming at characterizing the profile of high-risk pregnant women followed-up at specialized public outpatient clinics, as well as to verify and discuss aspects related to collaborative care in the decision on the route of birth delivery during the specialized prenatal care. The sample was calculated considering the prevalence of the high-risk pregnancy, morbidity profile and coverage of specialized services. Questionnaires were applied to 405 pregnant women of these services, and a descriptive analysis was performed based on the frequency distribution of the questions. The profile of pregnant women is mainly composed of young, black women, who have completed high school and without health insurance. There was a statistical association between variables: when women choose their own route of birth individually, the majority opts for normal birth; when only the doctor decides, the majority indicates cesarean 
section; and, when a joint decision is made the cesarean section prevails, but, in a lower percentage than when the doctor decides alone. It is necessary to discuss the power asymmetry between doctors and users in the care management as a fundamental component to qualify health work.

KEYWORDS Prenatal care. Pregnancy high-risk. Comprehensive health care. Decision making. Personal autonomy.

\section{Introdução}

A melhoria da saúde das gestantes foi elencada como um dos Objetivos de Desenvolvimento do Milênio (ODM), estabelecidos pela Organização Mundial da Saúde (OMS) no ano 2000, com o apoio de 191 nações $\mathbf{1}$. As metas estabelecidas para serem alcançadas até o ano de 2015 melhoraram indicadores relacionados à saúde em muitos países, inclusive das gestantes. A estratégia de traçar metas foi avaliada como positiva pela organização internacional, e, dando seguimento a suas ações propositivas, em 2015, foi elaborada a agenda 'Transformando o Nosso Mundo: A Agenda 2030 para o Desenvolvimento Sustentável'2, constituída por 17 Objetivos de Desenvolvimento Sustentável (ODS), para serem alcançados até 2030. Neste sentido, reconfigurando o quadro de preocupações mundiais, a saúde materna consta no objetivo número três dos ODS, denominado saúde e bem-estar, e é composta por nove metas a serem atingidas até 2030 , sendo que a primeira delas é a redução da taxa de mortalidade materna global para menos de 70 mortes por 100.000 nascidos vivos ${ }^{2}$.

No Brasil, as políticas de saúde que contemplam a saúde materno-infantil apresentam, desde 1999, a vigilância epidemiológica da mortalidade infantil e materna como responsabilidade do município ${ }^{3}$, e, junto a outras políticas, vêm implementando ações a fim de melhorarem os indicadores nacionais, o que contempla os objetivos de desenvolvimento apresentados anteriormente. Para fortalecer tais ações, em 2004, o Ministério da Saúde propôs um Pacto Nacional pela Redução da Mortalidade Materna e Neonatal ${ }^{4}$. O Pacto apresenta o objetivo de articular vários setores governamentais e sociedade civil para qualificar a atenção à saúde das mulheres e de recém-nascidos e afirma como meta a redução de 15\% da mortalidade materna e neonatal no País.

Conforme dados globais publicados em $2018^{5}$, entre 1990 e 2015 , a mortalidade materna no mundo caiu cerca de $44 \%$. No entanto, os índices de mortalidade materna permanecem entre os menos equitativos de todos os indicadores de saúde, que variam de menos de cinco mortes maternas por 100.000 nascidos vivos em países de alta renda para mais de 500 por 100.000 nascidos vivos em vários países da África Subsaariana ${ }^{6}$. O alto número de mortes maternas em algumas áreas do mundo reflete desigualdades no acesso aos serviços de saúde e destaca a lacuna entre ricos e pobres, sendo um índice sensível para inequidades sociais. Quase todas as mortes maternas ocorrem em países em desenvolvimento, e mais da metade ocorrem em ambientes frágeis e em contextos de crises humanitárias. Destacam-se, ainda, as disparidades entre mulheres com baixas e altas rendas e entre a população rural e a urbana ${ }^{5}$. 
A redução da mortalidade materna é central nas políticas de saúde pautadas na formação de Redes de Atenção à Saúde (RAS), que são arranjos organizativos de ações e serviços de saúde, de diferentes densidades tecnológicas, que, integradas por meio de sistemas de apoio técnico, logística e de gestão, buscam garantir a integralidade do cuidado ${ }^{7}$. Os pontos de atenção à saúde são espaços onde se ofertam determinados serviços de saúde de forma singular, cabendo às Unidades Básicas de Saúde (UBS) o papel de porta de entrada nas RAS.

No caso da redução da mortalidade materna, a Rede Cegonha ${ }^{8}$, instituída pelo Ministério da Saúde, em 2011, ao ser implementada em alguns estados, trouxe grandes avanços. Assim, são objetivos da Rede Cegonha: o acolhimento às intercorrências na gestação, com avaliação e classificação de risco e vulnerabilidade; acesso ao pré-natal de alto de risco em tempo oportuno; realização dos exames de pré-natal de risco habitual e de alto risco; e acesso aos resultados em tempo oportuno. Enfatiza-se que a Rede Cegonha, em relação às iniciativas anteriores de melhoria da atenção à saúde materno-infantil, traz como diferencial a articulação dos pontos de atenção numa rede de cuidados integral.

A mortalidade materna no Brasil caiu 58\% entre 1990 e 2015, passando de 143 para 60 óbitos maternos por 100 mil nascidos vivos ${ }^{9}$. As causas maternas caíram em $56 \%$, as neonatais em $56,7 \%$, e as nutricionais em 50,2\%. Em Campinas, no ano de 2016, a razão de mortalidade materna era de 33 óbitos para cada 100.000 nascidos vivos10, índice que supera a média nacional e alcança o Objetivo 5 do Desenvolvimento do Milênio de melhorar a saúde materna, com meta de 35 óbitos por 100 mil nascidos vivos em 2015.

No que se refere à saúde da gestante, o cuidado na gestação de alto risco é um dos aspectos essenciais a fim de reduzir a morbimortalidade infantil e materna. Considera-se gestação de alto risco

aquela na qual a vida ou a saúde da mãe e/ou do feto e/ou do recém-nascido têm maiores chances de serem atingidas que as da média da população considerada"1.

Os fatores que aumentam o risco de uma gestação podem ser divididos em fatores presentes antes da gestação e fatores que surgem durante a gestação.

Os fatores de risco presentes anteriormente à gestação podem estar relacionados às características individuais e condições sociodemográficas desfavoráveis, à história reprodutiva anterior ou às condições clínicas preexistentes. Já aqueles que surgem durante a gestação correspondem à exposição indevida ou acidental a fatores teratogênicos, doenças obstétricas na gravidez atual e intercorrências clínicas"1.

O objetivo de se determinar o risco de uma gestação é desencadear ações que possam reduzir a morbimortalidade infantil e materna, consideradas altamente evitáveis. Em geral, as gestantes de alto risco são encaminhadas para acompanhamento em um serviço especializado, contudo, recomenda-se que a gestante mantenha o vínculo com a equipe de atenção primária que iniciou o pré-natal"1, tal qual a proposta das RAS, incluindo a Rede Cegonha.

A atenção pré-natal para as mulheres com gestação de alto risco guarda características e protocolos específicos, inclusive orientações sobre o tipo de parto mais seguro em cada situação"1. De acordo com o 'Manual técnico de gestação de alto risco', a decisão deve ser tomada de acordo com cada caso, e é fundamental o esclarecimento da gestante e de sua família, cuja participação deve estar garantida no processo decisório. Salienta-se que gestação de risco não é sinônimo de cesariana, sendo possível a indução do parto ou aguardar o seu início espontâneo"11. Contudo, o que se tem evidenciado nos indicadores nacionais é o aumento no número de cesáreas, incluindo os casos de gestação de alto risco.

Há evidências de que, quanto mais as taxas de cesárea se distanciam dos $15 \%$ preconizados pela OMS, mais forte fica sua associação com a mortalida-de materna e com a mortalidade neonatal. No ano de $2007,47 \%$ de todos os partos 
ocorridos no País foram por cesariana, sendo que quase metade delas foi programada ${ }^{12}$. Em 2016, o Brasil figurava como vice-líder mundial em número de cesáreas, contabilizando 55,6\% dos partos no País. Em Campinas, a proporção de parto normal de mães residentes no município no ano de 2016 foi de $36,2 \%$, somados aqueles ocorridos no Sistema Único de Saúde (SUS) e na saúde suplementar ${ }^{13}$.

Em fevereiro de 2017, foi publicada a Portaria ${ }^{0} 353$ da Secretaria de Atenção à Saúde do Ministério da Saúde, que aprovou as Diretrizes Nacionais de Assistência ao Parto Normal, considerando que, apesar de o avanço da obstetrícia ter contribuído para melhorar os indicadores de morbidade e mortalidade materna e perinatais, tem-se hoje um modelo de assistência obstétrica que considera a gravidez, o parto e o nascimento como doenças e que submetem as gestantes e os recém-nascidos a altas taxas de intervenções. Essas diretrizes devem ser consideradas conjuntamente às Diretrizes de Atenção à Gestante: a operação Cesariana, aprovadas pela Portaria $\mathrm{n}^{0} 306$ e publicadas em 29 de março de 2016.

\section{Métodos}

Estudo transversal realizado com dados de inquérito de serviços que entrevistou 1.886 mulheres nos municípios de Campinas, Fortaleza, São Paulo e Porto Alegre, entre março e setembro de 2016. Este estudo foi realizado com os registros das gestantes de serviços especializados em gestação de alto risco do SUS Campinas. Foi selecionada uma amostra não probabilística, por serem reduzidos os números de serviços especializados e de pacientes atendidas nesses serviços. $\mathrm{O}$ número de pessoas para compor a amostra foi calculado pela expressão algébrica que determina o tamanho de amostras para estimação de proporções. Para uma proporção de 0,50 , correspondente à máxima variabilidade para a frequência dos eventos estudados, coeficiente de confiança de $95 \%(z=1,96)$, erro de amostragem de 5\%, o tamanho calculado da amostra foi de 400 gestantes.

Desta forma, foram entrevistadas todas as gestantes atendidas entre março e setembro de 2016 ( $n=405)$, em todos os serviços selecionados, considerando-se como população de estudo todas as pacientes residentes em Campinas e atendidas em consultas médicas nas unidades de saúde de atenção especializada e encaminhadas pela atenção básica que desejaram participar da pesquisa (critérios de inclusão).

Esta pesquisa foi aprovada pelo Comitê de Ética em Pesquisa da Unicamp sob o parecer $\mathrm{n}^{\mathrm{o}} 1.777 .800 / 2016$. Antes da aplicação de cada questionário, foi realizada a leitura do termo de consentimento livre e esclarecido, assinado por todas as mulheres que concordaram em participar do estudo, conforme Resolução $\mathrm{n}^{0}$ 466/2012.

As informações foram obtidas por meio de questionário estruturado previamente testado em estudo piloto e aplicado por entrevistadores treinados e supervisionados. Neste estudo, as seguintes variáveis foram consideradas:

Sociodemográficas e informações sobre atenção especializada: faixa etária (14 a 19, 20 a 29, 30 a 39, 40 anos e mais), cor da pele/raça (branca, preta, amarela, parda ou indígena), escolaridade (Ensino Fundamental, Ensino Médio ou Ensino Superior), posse de convênio/plano de saúde (sim ou não). Também, o tempo de gestação no momento da resposta ao questionário $\left(1^{\circ}, 2^{\circ}\right.$ ou $3^{\circ}$ trimestre), tempo de gestação no início do pré-natal na outra unidade que realizou o encaminhamento $\left(1^{\circ}\right.$, $2^{\circ}$ ou $3^{\circ}$ trimestre) e agendamento de retorno a partir da primeira consulta (sim ou não);

Cuidado compartilhado: foi feita a escolha da forma de parto (não; sim, parto normal; sim, parto cesáreo). Entre as que responderam 'sim', perguntou-se sobre a possibilidade de negociar com o médico a forma do parto (não, o médico escolheu sozinho; não, eu escolhi sozinha; sim). 
Foi realizada análise descritiva das variáveis selecionadas no estudo a partir da distribuição de frequências absolutas e relativas. Verificou-se a associação entre a forma de parto e o tempo de gestação. Ainda, foi verificada associação entre a possibilidade de decidir junto com o médico a forma do parto com a escolaridade, a cor da pele/raça da gestante e a forma de parto escolhida (cesárea ou normal). Essas análises foram realizadas pelo teste Quiquadrado de Pearson, considerando-se um nível de significância de $5 \%$.

\section{Local do estudo}

O município de Campinas é considerado de grande porte, com mais de um milhão de habitantes, sendo a cidade mais importante da Região Metropolitana de que faz parte. Está situada em um grande centro de desenvolvimento da indústria, dos agronegócios, de comércio e serviços do estado de São Paulo, além de ser conhecida nacionalmente como polo científico e tecnológico, contando com duas grandes universidades e diversas instituições de fomento à tecnologia ${ }^{\mathbf{1 4}}$.

Em Campinas, o processo de implantação do SUS desdobrou-se numa extensa e complexa rede de serviços, instâncias de gestão e controle social. Atualmente, a cidade é gestora plena de seu sistema de saúde, modalidade de gestão em que todas as decisões quanto ao gerenciamento de recursos e serviços, sejam eles próprios, conveniados ou contratados, dão-se no âmbito do município.

No caso da gestação de alto risco, os pontos de atenção à saúde das gestantes são as UBS, ambulatórios especializados em pré-natal de risco, maternidades e hospitais. Em Campinas, há 63 UBS e 163 equipes de saúde da famíliaº. Por ocasião da implantação do Programa de Saúde da Família de Campinas/Paideia, em 2001, decidiu-se trabalhar com equipes ampliadas, mantendo ginecologistas e pediatras, juntamente com médicos generalistas, nas equipes de saúde da família, de forma que cada equipe se responsabilizasse por cerca de 1.400 famílias ou 7.000 pessoas. Assim, o pré-natal na UBS pode ser realizado tanto por médicos generalistas quanto por ginecologistas.

Frente a uma gestante considerada de alto risco, a equipe da UBS pode encaminhá-la ao Centro de Atenção Integrada à Saúde da Mulher (Caism), da Unicamp, ao Hospital da Pontifícia Universidade Católica de Campinas e à Maternidade de Campinas, por meio de um sistema informatizado de regulação (Sistema On-line, o SOL), a partir de critérios de encaminhamento definidos segundo o protocolo municipal ${ }^{15}$.

\section{Resultados e discussão}

Entre as gestantes entrevistadas, a média de idade foi de 32,1 anos $(\mathrm{dp}=2,1)$, sendo que $16,1 \%$ eram menores de 20 ou maiores de 40 anos no momento da aplicação do questionário. Esses limites etários fazem parte do conjunto de critérios presentes no protocolo de encaminhamento ao pré-natal de alto risco do município. Quanto à cor da pele/raça, 42,8\% se declararam pardas. Com relação à escolaridade, a maior parte cursou o Ensino Médio, $50,7 \%$, e apenas $8,9 \%$ iniciaram ou concluíram o Ensino Superior. Quase 90,0\% das gestantes não possuíam convênio/plano de saúde, conforme mostrado na tabela 1.

No que se refere ao tempo atual de gestação, $59,9 \%$ estavam no $3^{\circ}$ trimestre na ocasião da aplicação do questionário, e a maioria, 85,6\%, iniciou o pré-natal ainda no $1^{\circ}$ trimestre de gestação. Em torno de $97,0 \%$ das gestantes saíram da primeira consulta do pré-natal de risco com a consulta de retorno agendada (tabela 1). 
Tabela 1. Características sociodemográficas das entrevistadas e informações sobre atenção especializada. Campinas, 2016

\begin{tabular}{|c|c|c|}
\hline Variáveis/categorias & $\mathrm{n}$ & $\%$ \\
\hline \multicolumn{3}{|l|}{ Faixa etária } \\
\hline 14 a 19 & 32 & 7,9 \\
\hline 20 a 29 & 169 & 41,8 \\
\hline 30 a 39 & 170 & 42,1 \\
\hline 40 e mais & 33 & 8,2 \\
\hline \multicolumn{3}{|l|}{ Cor da pele } \\
\hline Branca & 149 & 37,1 \\
\hline Parda & 172 & 42,8 \\
\hline Negra/Preta & 74 & 18,4 \\
\hline Outras & 7 & 1,7 \\
\hline \multicolumn{3}{|l|}{ Escolaridade } \\
\hline Analfabeto + Ensino Fundamental incompleto & 23 & 21,5 \\
\hline Ensino Fundamental completo & 14 & 5,7 \\
\hline Ensino Médio incompleto & 53 & 13,2 \\
\hline Ensino Médio completo & 205 & 50,7 \\
\hline Superior incompleto & 14 & 3,5 \\
\hline Superior completo & 22 & 5,4 \\
\hline \multicolumn{3}{|l|}{ Posse de convênio/plano de saúde } \\
\hline Não & 363 & 89,8 \\
\hline Sim & 41 & 10,2 \\
\hline \multicolumn{3}{|l|}{ Tempo de gestação atual } \\
\hline 10 trimestre & 27 & 6,7 \\
\hline 20 trimestre & 135 & 33,4 \\
\hline 3 trimestre & 242 & 59,9 \\
\hline \multicolumn{3}{|c|}{ Tempo de gestação no início do pré-natal na outra Unidade que realizou o encaminhamento } \\
\hline 10 trimestre & 345 & 85,6 \\
\hline 20 trimestre & 48 & 11,9 \\
\hline 3으 trimestre & 10 & 2,5 \\
\hline \multicolumn{3}{|c|}{ Deixou a primeira consulta do pré-natal de risco com agendamento para retorno } \\
\hline Não & 12 & 3,0 \\
\hline Sim & 391 & 97,0 \\
\hline
\end{tabular}

Fonte: Elaboração própria.

O início precoce do pré-natal, tal qual preconizado pela Rede Cegonha ${ }^{8}$ em 2013, demonstra que as gestantes atendidas pelo SUS Campinas não encontraram barreiras de acesso importantes, como sabido em outras cidades do País ${ }^{16,17}$. Pode-se supor que a alta cobertura de serviços da atenção primária e a presença de médicos ginecologista-obstetras 
na atenção básica do município contribuíram positivamente para a captação das gestantes em tempo oportuno.

Por outro lado, ainda que existam critérios reunidos em um protocolo municipal15 para encaminhamento das gestantes ao Pré-Natal de Alto Risco (PNAR), verificou-se que 49,4\% das gestantes foram inseridas nos serviços especializados ainda no primeiro trimestre da gestação. Considerando a existência de profissionais especialistas na atenção básica e os critérios elencados no protocolo de encaminhamento ao PNAR, chama a atenção que tantas mulheres sejam inseridas precocemente em serviço especializado, uma vez que a maior parte das doenças obstétricas relevantes ocorre a partir do segundo trimestre de gestação ${ }^{18}$. Ainda assim, poderiam estar sob cuidados em seu território com o médico gineco-obstetra presente no modelo de atenção básica adotado pelo município.

No caso de a consulta de retorno estar garantida a quase todas as gestantes que se consultaram pela primeira vez no serviço especializado, embora, em uma primeira leitura, possa parecer positivo, cabe refletir se há uma inadequada fixação de gestantes no serviço especializado que poderiam seguir o pré-natal em seu bairro, na unidade básica de saúde, com as devidas orientações a partir do diagnóstico feito pelo serviço especializado.

Nos gráficos 1 e 2, estão apresentadas algumas variáveis relacionadas à decisão compartilhada sobre a via do parto. Observou-se que a maioria das mulheres não havia conversado com o médico sobre o tipo de parto, e, das que responderam positivamente, apenas $35,5 \%$ escolheram junto ao médico.

Gráfico 1. Possibilidade de negociar com o médico a via do parto. Campinas, 2016

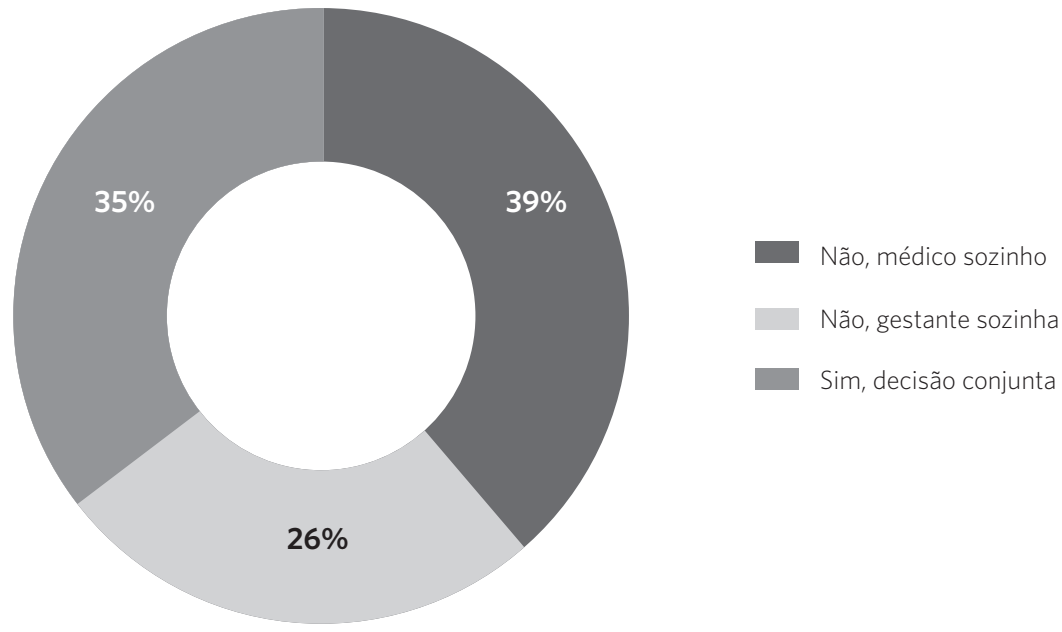

Fonte: Elaboração própria.

No que se refere à escolha da forma de parto e ao tempo de gestação, das que responderam $(n=391), 66,8 \%$ ainda não haviam escolhido, e, entre essas, apenas 8,0\% estavam no primeiro trimestre de gestação. Destacase, ainda, que, entre as mulheres no terceiro trimestre de gestação, 65,2\% $(\mathrm{n}=145)$ também não fizerem referência à escolha do tipo de parto (normal ou cesárea). Por fim, não houve associação estatisticamente significativa entre a forma do parto escolhida e o tempo de gestação $(p=0,113)$. 
Gráfico 2. Decisão da forma de parto. Campinas, 2016

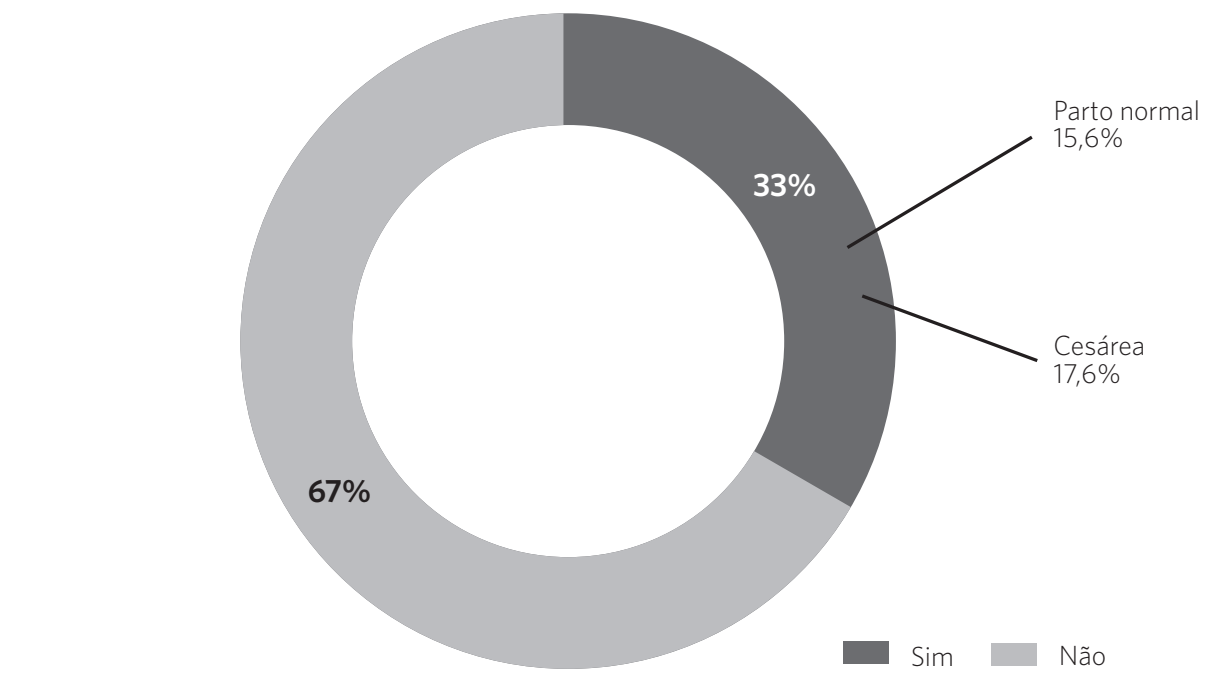

Fonte: Elaboração própria.

Destaca-se, ainda, que, entre as mulheres que estavam no terceiro trimestre de gestação, $65,2 \%(n=145)$ também não fizeram referência à escolha do tipo de parto (normal ou cesárea). Por fim, não houve associação estatisticamente significativa entre a forma do parto escolhida e o tempo de gestação $(\mathrm{p}=0,113)$.

Nota-se que a temática do parto propriamente dito está pouco presente nas consultas de pré-natal, mesmo nos estágios avançados da gestação. Outro estudo ${ }^{18}$ aponta, ainda, que as orientações sobre práticas para facilitar o parto apresentaram baixa frequência, reforçando o caráter biomédico da assistência.

Em 2018, a OMS publicou'19 um conjunto de recomendações para garantir que grávidas saudáveis tenham uma experiência positiva na hora do parto natural, objetivando reduzir intervenções médicas desnecessárias. Entre as orientações, inclui-se uma melhor comunicação entre os médicos e as mães, permitindo que as mulheres também possam opinar sobre a administração de sua dor durante o processo de dilatação e posições para o parto. Tradicionalmente, os serviços de saúde têm uma tendência a identificar o usuário como um ser passivo, alijado de recursos para lidar com sua situação, de modo a reforçar sua dependência e identificar a potência apenas no polo do profissional.

Destaca-se que, embora um percentual significativo das mulheres tenha feito a escolha da via do parto sem discutir com o médico, entende-se que essa não é uma escolha autônoma ou reveladora de protagonismo, uma vez que o estudo investigou se 'foi possível negociar a forma de parto'. Ou seja, essas mulheres podem não ter recebido a atenção necessária para construir sua decisão, apoiadas em informações técnicas do médico e com possibilidade de lhe comunicar seus desejos e anseios pessoais dentro de uma relação de cuidado compartilhado e confiança. Chama, ainda, a atenção o relato de que $39 \%$ das decisões foram tomadas exclusivamente pelo médico, o que evidencia a impossibilidade de diálogo sobre a forma de parto.

Para as gestantes que referiram ter sido feita a escolha do tipo de parto (normal ou cesárea, $\mathrm{n}=121$ ), foi verificada a associação com a possibilidade de decidir com o médico. Entre as mulheres que relataram escolha individual do médico, 71,7\% dos profissionais optaram pela cesárea, e, na escolha individual da 
*Orcid (Open Researcher and Contributor ID). gestante, $84,4 \%$ optaram pelo parto normal. Já entre as que mencionaram a possibilidade de decisão conjunta, $60,5 \%$ optaram pela cesárea. Houve diferença estatisticamente significativa $(\mathrm{p}<0,001)$ em relação à forma de parto e a possibilidade de escolha (dados não apresentados em tabela).

Esses resultados apontam para situação semelhante ao estudo de Barbosa sobre parto cesáreo ${ }^{20}$, em que apenas $17 \%$ das mulheres entrevistadas solicitaram cesárea. Conforme a análise de Tedesco ${ }^{21}$, em geral, a gestante não participa da escolha da via de parto, sendo apenas informada sobre a decisão. Essa realidade tem sido apontada como um dos fatores que contribuem para o aumento do parto cirúrgico. Além disso, ressaltamos que, para a escolha individual do médico ou para a possibilidade de decisão conjunta, em que o tipo de parto foi a cesárea, $62,7 \%$ das mulheres $(n=59)$ não souberam dizer o motivo da escolha.

Não foi verificada associação estatística ( $p>0,05)$ para escolaridade e cor da pele/ raça da gestante quanto à possibilidade de escolha. Contudo, outros estudos realizados no País encontraram relevantes relações entre desigualdades raciais e sociodemográficas e a assistência ao pré-natal e ao parto ${ }^{22,23}$.

Por fim, destaca-se que os acontecimentos são sempre coproduzidos, bem como os sujeitos e as organizações, de forma que há uma corresponsabilidade pela constituição de contextos singulares ${ }^{24}$. Admitir que os sujeitos e fatores estão em constante interação, influenciando-se mutuamente, é ponto de partida para reflexão e mudanças nas práticas do trabalho em saúde. Para o desenvolvimento de uma relação de cuidado integral, é necessário incluir a centralidade da participação do usuário e considerá-lo sujeito de sua vida em todos os momentos, inclusive nas circunstâncias de fragilidade psíquica e biológica. Ao destacar o papel ativo dos sujeitos na coprodução de saúde, reafirma-se o seu protagonismo na gestão de si e na relação com suas próprias limitações.

\section{Considerações finais}

Foi encontrado um perfil de gestantes majoritariamente jovens, negras, com ensino fundamental completo e sem plano de saúde nos serviços especializados em gestação de alto risco de Campinas.

Os testes estatísticos demonstram associação entre as variáveis 'tipo de parto escolhido' e 'possibilidade de escolher conjuntamente o tipo de parto'. Quando as mulheres fazem a escolha do seu parto individualmente, a maioria delas opta pelo parto normal. Por outro lado, quando o médico decide sozinho sobre o tipo de parto a ser realizado, a maioria indica a cesariana. Nos casos em que foi possível fazer a cogestão da decisão sobre o tipo de parto a ser escolhido, verificou-se que prevalece a opção pela cesariana, porém, em percentual bem mais baixo do que quando o médico decide sozinho.

Apontamos para a necessidade de discutir a assimetria de poder entre médicos e gestantes de alto risco na cogestão do cuidado. Este estudo limitou-se a verificar associações estatísticas para as características consideradas, sendo necessárias pesquisas qualitativas para ajudar a elucidar essa relação, especialmente por meio de observação e entrevistas em profundidade com ambos os atores.

\section{Colaboradores}

Fernandes JA (0000-0001-6057-5058)* contribuiu substancialmente para a concepção e para a análise e a interpretação dos dados; contribuiu significativamente para a elaboração do rascunho e participou da aprovação da versão final do manuscrito. Campos GWS (0000-0001-5195-0215)* contribuiu significativamente para a revisão crítica do conteúdo e participou da aprovação da versão final do manuscrito. Francisco PMSB (0000-00017361-9961)* contribuiu para a análise e a interpretação dos dados e participou da aprovação da versão final do manuscrito. 


\section{Referências}

1. Organização Mundial da Saúde. Objetivos de Desenvolvimento do Milênio Brasil [internet]. [acesso em 2019 fev 2]. Disponível em: http://www.odmbrasil. gov.br/.

2. Programa das Nações Unidas para o Desenvolvimento. Acompanhando a agenda 2030 para o desenvolvimento sustentável: subsídios iniciais do Sistema das Nações Unidas no Brasil sobre a identificação de indicadores nacionais referentes aos objetivos de desenvolvimento sustentável [internet]. Brasília, DF: PNUD; 2015 [acesso em 2019 fev 2]. Disponível em: http://www.undp.org/content/dam/brazil/docs/ agenda2030/undp-br-Acompanhando-Agenda2030-Subsidios_iniciais-Brasil-2016.pdf.

3. Brasil. Ministério da Saúde. Manual de vigilância do óbito infantil e fetal e do Comitê de Prevenção do Óbito Infantil e Fetal. Brasília, DF: MS; 2009.

4. Brasil. Ministério da Saúde, Secretaria de Atenção à Saúde, Departamento de Ações Programáticas Estratégicas. Pacto Nacional pela Redução da Mortalidade Materna e Neonatal [internet]. Brasília, DF: MS; 2004 [acesso em 2019 fev 2]. Disponível em: http://portalarquivos.saude.gov.br/ images/pdf/2016/junho/20/2.a\%20Pacto\%20 redu\%C3\%A7\%C3\%A30\%20mortalidade.pdf.

5. Organização Pan-Americana da Saúde. Folha informativa sobre mortalidade materna. [internet]. Brasília, DF: Opas; 2018 [acesso em 2019 fev 2]. Disponível em: https:/www.paho.org/bra/index.php?option=com content\&view=article\&id=5741: folha-informativa-mortalidade-materna $\&$ Itemid $=820$.

6. Restrepo-Méndez MC, Victora CG. Maternal mortality by age: who is most at risk? Lancet. 2014; 2(3):120121.

7. Santos L. Região de saúde e suas redes de atenção: modelo organizativo-sistêmico do SUS. Ciênc. Saúde Colet. 2017; 22(4): 1281-1289.

8. Brasil. Ministério da Saúde. Portaria nº 1459, de 24 de junho de 2011. Institui no âmbito do Sistema Único de Saúde - SUS - a Rede Cegonha. Diário Oficial da União. 24 Jun 2011.

9. Souza MFM, Malta DC, Franca EB, et al. Transição da saúde e da doença no Brasil e nas Unidades Federadas durante os 30 anos do Sistema Único de Saúde. Ciênc. Saúde Colet. [internet]. 2018 [acesso em 2018 jul 18]; 23(6):1737-1750. Disponível em: http:// ref.scielo.org/p6zsb4.

10. Secretaria Municipal de Saúde [internet]. Campinas: Prefeitura Municipal de Campinas; 2016. [acesso em 2017 maio 5]. Disponível em: http://www.saude.campinas.sp.gov.br/saude/.

11. Brasil. Ministério da Saúde. Gestação de Alto Risco: Manual Técnico. Brasília, DF: MS; 2012.

12. Victora CG, Aquino EM, Leal MC, et al. Maternal and child health in Brazil: progress and challenges. Lancet. 2011; 377(9780):1863-76.

13. Secretaria Municipal de Saúde. Plano Municipal de Saúde de Campinas 2018-2021. [internet]. Campinas: Prefeitura Municipal de Campinas; 2017 [acesso em 2017 maio 10]. Disponível em: http://www.saude.campinas.sp.gov.br/cms/textos/2017/PMS_2018_2021_e_ PAS_2018_para_aprovacao.pdf.

14. Fernandes JA. Apoio institucional e cogestão: uma reflexão sobre o trabalho dos apoiadores do SUS Campinas [dissertação] [internet]. Campinas: Universidade Estadual de Campinas; 2014. 100 p. [acesso em 2019 jun 3]. Disponível em: http://repositorio.unicamp.br/bitstream/REPOSIP/312912/1/Fernandes_ JulianaAzevedo_M.pdf .

15. Secretaria Municipal de Saúde. Protocolo Pré-Natal de Alto Risco. [internet]. Campinas: Prefeitura Municipal de Campinas; 2016 [acesso em $2016 \mathrm{dez}$ 10]. Disponível em: http://www.campinas.sp.gov.br/governo/saude/atencao-a saude/saudeDaMulher_protocolos_espec_preNatalRisco.php. 
16. Fernandes RZS, Vilela MFG. Estratégias de integração das práticas assistenciais de saúde e de vigilância sanitária no contexto de implementação de Rede Cegonha. Ciênc. Saúde Colet. 2014; 19(11):4457-4466.

17. Viellas EF, Domingues RMSM, Dias MAB et al. Assistência pré-natal no Brasil. Cad. Saúde Pública. 2014; 30(1):85-100.

18. Moura BLA, Alencar GP, Silva ZP, et al. Internações por complicações obstétricas na gestação e desfechos maternos e perinatais, em uma coorte de gestantes no Sistema Único de Saúde no Município de São Paulo. Cad. Saúde Pública [internet]. 2018 [acesso 2018 fev 17]; 34(01):1-13. Disponível em: http://www.scielo.br/ pdf/csp/v34nl/1678-4464-csp-34-01-e00188016.pdf.

19. World Health Organization. Who Recommendations: intrapartum care for a positive childbirth experience [internet]. Geneva: World Health Organization; 2018 [acesso em 2018 fev 17]. Disponível em: https://www. who.int/reproductivehealth/publications/intrapartum-care-guidelines/en/.

20. Barbosa GP, Giffin K, Angulo-Tuesta A, et al. Parto cesáreo: quem o deseja? Em quais circunstâncias? Cad. Saúde Pública. 2003; 19(6):1611-1620.
21. Tedesco RP, Maia Filho NL, Mathias L et. al. Fatores determinantes para as expectativas de primigestas acerca da via de parto. Rev. bras. ginecol. obstet. 2004; 26(10):791-798.

22. Leal MC, Gama SGN, Cunha CB. Desigualdades raciais, sociodemográficas e na assistência ao pré-natal e ao parto, 1999-2001. Rev. Saúde Pública. 2005; 39(1):100-117.

23. Leal MC, Szwarcwald CL, Almeida PVB et al. Saúde reprodutiva, materna, neonatal e infantil nos 30 anos do Sistema Único de Saúde (SUS). Ciênc. Saúde Colet. 2018; 23(6):1915-1928.

24. Campos GWS, Onocko RT. Coconstrução de autonomia: o sujeito em questão. In: Campos GWS, Akerman M, Minayo MCS, organizadores. Tratado de Saúde Coletiva. São Paulo: Hucitec; 2012. p. 669-688.

\footnotetext{
Recebido em 19/07/2018

Aprovado em 17/02/2019

Conflito de interesses: inexistente

Suporte financeiro: Coordenação de Aperfeiçoamento de Pessoal de Nível Superior (Capes)
} 